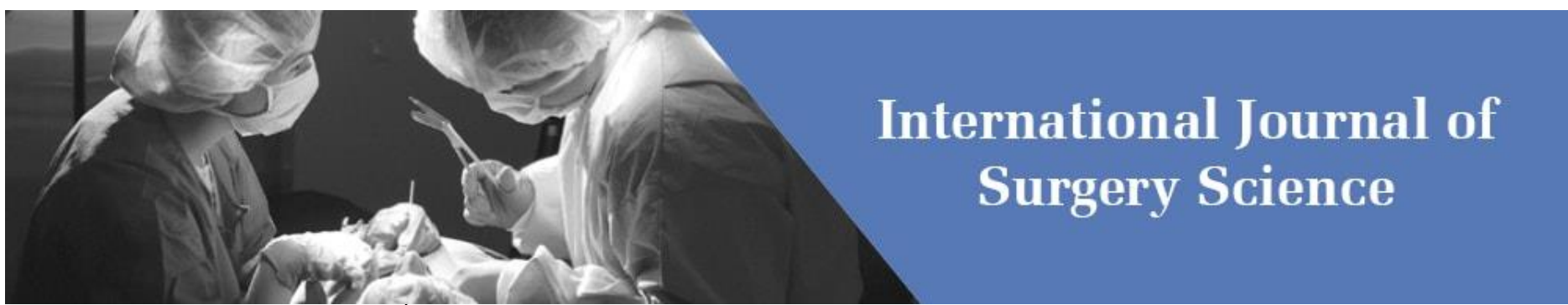

E-ISSN: 2616-3470

P-ISSN: 2616-3462

(C) Surgery Science

www.surgeryscience.com

2021; 5(2): 304-306

Received: 12-02-2021

Accepted: 16-03-2021

Dr. Mohammed R Shuber

Karbalaa Health Directorate,

Karbalaa, Iraq

Dr. Ihsan AL-Ghanimi

Karbalaa Health Directorate,

Karbalaa, Iraq
Corresponding Author:

Dr. Mohammed R Shuber

Karbalaa Health Directorate,

Karbalaa, Iraq

\section{Comparative study between internal and external osteotomy in rhinoplasty}

\author{
Dr. Mohammed R Shuber and Dr. Ihsan AL-Ghanimi
}

DOI: https://doi.org/10.33545/surgery.2021.v5.i2e.710

\section{Abstract}

Background: The aim of this study is to compare between internal osteotomy and external osteotomy in patients undergoing rhinoplasty.

Methods: The study involved 50 patients, 25 of them had external percutaneous lateral osteotomy, the other 25 patients had internal endonasal lateral osteotomy. The internal osteotomy was done low to high using $4 \mathrm{~mm}$. curved guarded osteotome. The external osteotomy was done low to low using $2 \mathrm{~mm}$ sharp osteotome. The grading system of Kara and Gokalan was followed for edema and ecchymosis.

Results: The mucosal tear is found in 4 patients of group - A (those who had external osteotomy).

(16\%), while 20 patients of group-B (those who had internal osteotomy) $(80 \%)$ had injury to nasal mucosa. In group-A patients only 5 patients $(20 \%)$ develop periorbital oedema, all were of Grade 2 . All the patients of group-B (100\%) develop periorbital edema, 2 patients (8\%) of grade 4 edema, 10 patients (40\%) of grade 3 edema, 12 patients $(48 \%)$ of grade 2 edema and one patient $(4 \%)$ of grade 1 edema. The ecchymosis of grade 1 was found in 3 patients of group-A (12\%) and in 20 patients of group-B (80\%).

Conclusion: External osteotomy is preferred over internal osteotomy with less complication rate. It causes much less mucosal injury and significant reduction in periorbital edema and ecchymosis without scar formation.

Keywords: internal, external, osteotomy

\section{Introduction}

Nasal pyramid consists of paired nasal bones attached laterally with frontal process of maxilla through naso-maxillary suture and superiorly with frontal bone through fronto-nasal suture and with each other in the mid line through inter-nasal suture. The bony septum is made of perpendicular plate of ethmoid superiorly and vomer inferiorly. The perpendicular plate is attached with nasal bones and vomer is attached to maxillary crest. The relationship between these bony attachments may be affected in nasal trauma. The nasal bones get support from two places first its base which is attached to frontal bone, second is from its side which is attached to frontal process of maxilla. Osteotomy is done for two purposes first is to reposition the base of the nose, second to reduce the convexity of lateral surface. Lateral osteotomy is specifically design on the thickness of bone which increase as we go laterally and superiorly, so every osteotomy is design to go through thinner parts as the cuts are more précised and need less force with less mucosal damage. The thicker part of the bone is corresponded to nasomaxillary suture, so never do osteotomy on a thicker part as this will produce a step deformity which unacceptable especially in a thin skin people. Osteotomy may be classified into medial, intermediate and lateral. Lateral osteotomy is of two types, low to high and low to low osteotomy. In low to high type (Figure:1) we start low to the junction of naso-maxillary suture to the pyriform aperture and then go up through thinner part until the inter-canthal line then we go medially, grossing the naso-maxillary suture. In low to low osteotomy (Figure:2) we start low and remain low going parallel to naso-maxillary suture into intercanthal line which is later joined by medial osteotomy. Sometime a little concave bone needs an intermediate osteotomy to joined the medial osteotomy. There are few basic principles of osteotomy first is always do interrupted cut line osteotomy as this will give a ragged edge which helped in rapid healing and give more stability. Interrupter line gives a controlled cut while a deep one disrupts the mucosa. Another thing is always keeping your osteotome sharp, use the smallest one, $2 \mathrm{~mm}$ osteotome is the best. Always keep osteotome angulated as this will required less force and produced an accurate cut. 
Another problem is bleeding which result from damaging small branches of angular artery. To avoid this vessel, whenever you do stab incision and you place the oteotome on skin, first move the soft tissue a little up and then grasp the bone and come down then do osteotomy cut. Lateral osteotomy should be done first then medial osteotomy. While doing lateral 0steotomy always start in the mid line then go down as bone is thicker down and then go cephalically to complete osteotomy while in medial osteotomy always start from medial aspect then com to join the low to low osteotomy. Lateral osteotomies usually delay to the end of surgery because of hemorrhage induced by fracturing the bones. It is a blind procedure, so there is always a risk of injuring supporting periosteum and mucosa. An ideal osteotomy should be precise, predictable, achieving adequate asthetic and functional results with minimum soft tissue damage.

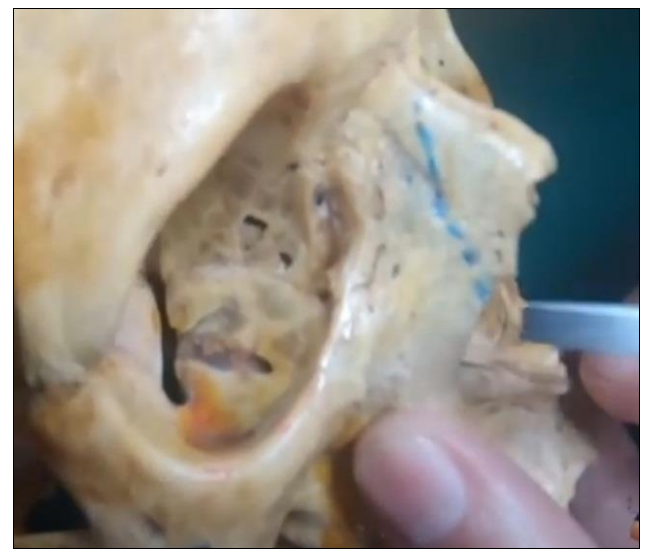

Fig 1: Low to high osteotomy

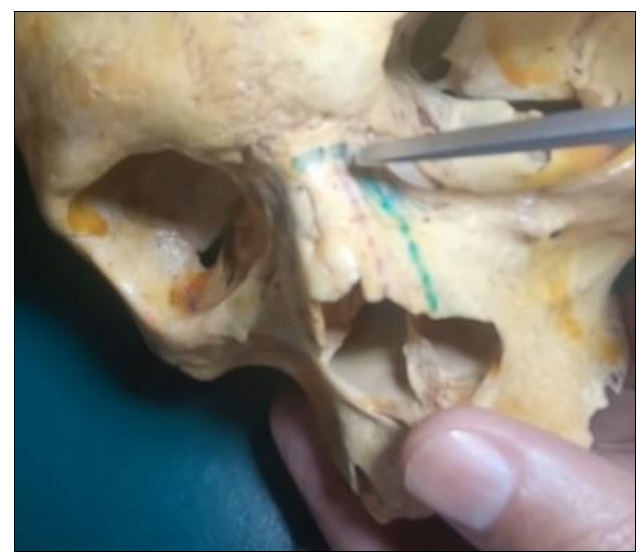

Fig 2: Low to low osteotomy

\section{Rationale}

Rhinoplasty is becoming a commonly performed surgery now a day and different techniques are developed to reduce the complications which include hemorrhage, peiorbital edema, ecchymosis, excessive damage to nasal mucosa, and asymmetry which may lead to aesthetic deformity and nasal obstruction. The lateral osteotomy is design to medialize the nasal bones and narrow the bridge of the nose. It can be done either by the internal endonasal technique or by external percutaneous technique. The choice of technique is depending on surgeon preference and practice. The rationale behind this study is to compare between these two techniques regarding results and complications.

\section{Method and Material}

A blind control study was done of total 50 patients who were operated for the rhinoplasty in Imam Zain AL-Abideen hospital in Karbalaa, Iraq. Data collected over two years period and patients were followed for six months after surgery. Patients were divided into two group, each of 25 patients. Group (A) underwent external lateral osteotomy, all done by first author. Group (B) underwent internal lateral osteotomy, all done by second author. All the patients had rhinoplasty under general anesthesia. The nose is injected with adrenaline 1:80,000 to reduce bleeding. Immediately after surgery all patients were examined by rigid nasoscope to assess mucosal damage. Periorbital oedema and ecchymosis were assessed in the ward when patients waked up using scoring system modified by Kara and Gokalan in $1999^{[10]}$, as follow:

\section{Grading system for periorbital oedem}

Grade 1 - No coverage of iris with eyelids

Grade 2 - Slight coverage of iris with swollen eyelids

Grade 3 - Full coverage of iris with swollen eyelids

Grade 4 - Full closure of eyes

\section{Grading system for ecchymosis}

Grade 1 - Eccyhmosis up to the medial 1/3 of lower and/or upper eyelid

Grade 2 - Ecchymosis up to the medial 2/3 of the lower and/or upper eyelid

Grade 3 - Ecchymosis involving whole length of upper and/or upper eyelid

\section{Operative procedure}

The patients in group (A) underwent external percutaneous low to low lateral osteotomy with $2 \mathrm{~mm}$. osteotome. The patients in group (B) underwent endonasal low to high continuous osteotomy. The proposed line of osteotomy was marked with marking pen for the accurate path of osteotomy. The endonasal lateral osteotomy was done by making a small incision at pyriform aperture just above the level of attachment of inferior turbinate. The osteotomy below this level may cause medicalization of inferior turbinate in long term with subsequent obstruction. Care should be taken to avoid damage to Webster's bony triangle which may alter the shape of vestibule. The periosteum was elevated to the root of the nose using periosteal elevator. The osteotome was engaged below the level of nasomaxillary suture and then passed along the nasofacial groove with tap stroke of the mallet. Light force is required first to engage the osteotome then more force is required on moving up. The force of taping is increased by increasing the distance between mallet and osteotome. The finger of the other hand should guide the tip of osteotome to avoid orbital injury. The osteotome was gently curved medially on approaching the infraorbital rim then superiorly to join the medial osteotomy. The pyriforme incision is closed at the end of surgery although it is not always required.

\section{Percutaneous perforatin osteotomy}

The sharp tip of $2 \mathrm{~mm}$ osteotome is applied at a midpoint between vestibule and medial canthus. This obviate making a stab incision since the tip of osteotome is too sharp to cut through a skin and subcutaneous tissue. The tip of osteotome was sweeping up and down to reach bone without elevation of periosteum or damaging lacrimal sac. Perforation is made at the proposed line of osteotomy with tap stroke of malate. A multiple perforation is required to fracture bone, this can be done by rotating the osteotome up and down from the same skin incision. Care should be taken to avoid excessive force as this may 
damage the underlying mucosa of the bone. When the sound of taping of osteotome is change this signals that internal cortical bone has been broken. In addition, giving way feeling indicate undue perforation of nasal mucosa. For transverse osteotomy, tip of osteotome is applied at a midpoint between canthus and nasion. The periosteum is incised with the tip of osteotome and perforating osteotomy is made in a similar manner. Slight digital pressure was required to fracture the nasal bones and achieve medicalization. There is no need to close skin incision as it heals rapidly without visible scar.

\section{Results}

The mucosal injury is found in 4 patients of group - A (those who had external osteotomy) (16\%), while 20 patients of group B (those who had internal osteotomy) (80\%) had mucosal injury at some site of the nose as detected by endoscopy. In group - A patients only 5 patients $(20 \%)$ develop periorbital oedema, all were of Grade 2. All the patients of group-B (100\%) had periorbital edema as follow:

2 patients $(8 \%)$ had grade - 4 edema.

10 patients $(40 \%)$ had grade -3 edema.

12 patients $(48 \%)$ had grade -2 edema.

1 patient (4\%) had grade -1 edema.

The ecchymosis of grade 1 was found in 3 patients of group-A $(12 \%)$ and in 20 patients of group - B $(80 \%)$.

Table 1: Internal and external osteotomy

\begin{tabular}{|c|c|c|}
\hline & External osteotomy & Internal osteotomy \\
\hline Mucosal injury & $16 \%$ & $80 \%$ \\
\hline Periorbital edema & $20 \%$ & $100 \%$ \\
\hline Ecchymosis & $12 \%$ & $80 \%$ \\
\hline
\end{tabular}

\section{Discussion}

The external approach was popularized by Straatsma ${ }^{[6]}$. This approach causes less damage to nasal mucosa with preservation of the supporting periosteum which lead to less postoperative complications and rapid recovery. In group-A patients (percutanous osteotomy), the periosteum was not elevated, instead sweeping movement up and down skin incision is don reaching the nasal bones directly. Only 4 patients developed mucosal tear, 5 patients developed edema of grade 2 and 3 patients developed ecchymosis of grade 1 . In group-B patients (internal osteotomy) all the 25 patients had edema and 20 patients had mucosal tear and ecchymosis. The use of steroids during the first 24 hour is helpful in reducing edema and has less effect on ecchymosis since the peak time of edema is the second post-operative day and it recover within 7 days. However, Vikas Sinha et al. performed a retrospective study on 45 patients and found that external osteotomy produces less edema, ecchymosis and mucosal tear. However, our results are consistent with him. Yucel ${ }^{[7]}$ in his study of external vs. internal osteotomy found that both techniques of osteotomy almost produce a similar result regarding edema although ecchymosis is less in internal osteotomy. However, our result was very different from him. Gryskiewicz JM and Gryskiewics KM ${ }^{[11]}$ carried out a similar study involving 50 patients undergoing lateral osteotomy (25 perforating lateral osteotomy and 25 continuous osteotomy) and found that perforating lateral osteotomy produce less ecchymosis and edema compared to continuous osteotomy. They found that external osteotomy is superior to internal osteotomy with less morbidity and produce more controlled fracture. Giacomarra et al. ${ }^{[9]}$ perform a more expanded study involving 142 cases of rhinopasty and concluded that external osteotomy is a superior approach in comparison with internal osteotomy. Our finding is very consistent with their findings. A complete cut through the entire thickness of nasal bone is not required and may increase the risk of mucosal injury, so micro fracture of the outer cortex of bone is enough and the remaining thickness of bone is fractured by digital pressure. After doing medial and lateral osteotomy mobilization of nasal bones become easy and nasal bridge can be narrows by approximating the nasal bones in the mid line. Some surgeon prefers to lift a sub-periosteal tunnel prior to osteotomy to avoid injuring the angular artery but lifting this tunnel increase the risk of injuring the lacrimal sac and medial canthal ligament, so it remains a subject of debate.

In external osteotomy the bleeding is less and this means less edema and minimal mucosal injury and no scars is seen during the whole follow up period.

\section{Conclusion}

External osteotomy is preferable over internal osteotmy as it make a green stick fracture so the hump remain stable with less mucosal injury. The periorbital edema and ecchymosis are much less than internal osteotomy with no visible scar formation.

\section{Acknowledgment}

The authors present this study to spirits of our colleagues who died during corona pandemic.

\section{References}

1. Lee HM, Kang HJ, Choi JH, Chae SW, Lee SH, Hwang SJ. Rationale for osteotome selection in rhinoplasty. The Journal of Laryngology \& Otology 2002;116:1005-1008.

2. Tardy Jr. MA, Denneney JC. Micro-osteotomies in rhinoplasty. Facial Plast Surg 1984;1:137-41. (Cross reference).

3. Rohrich RJ, Krueger JK, Adams WP Jr, Hollier LH Jr. Achieving consistency in the lateral nasal osteotomy during rhinoplasty: An external perforated technique. Plast. Reconstr. Surg 2001;108:2122. (Cross reference).

4. Becker DG, McLaughlin Jr, Loevner LA, Mang A. The lateral osteotomy in rhinoplasty: Clinical and radiolographic rationale for osteotome selection. Plastic Reconstructive Surgery 2000;105:1806-16.

5. Denecke HJ, Meyer R. Plastic Surgery of the Head and Neck: Corrective and reconstructive Rhinoplasty. New York: Springer-Verlag 1967. (Cross reference).

6. Straatsma CR. Surgery of the bony nose: Comparative evaluation of chisel and saw technique. Plastic reconstructive surgery 1961;28:246. (Cross reference).

7. Yucel OT. Which type of osteotomy for edema and ecchymosis external or internal. Annals of Plastic Surgery 2005;55(6)587-590.

8. Rohrich RJ, Janis JE, Adams WP, Krueger JK. An update on the lateral nasal osteotomy in rhinoplasty; an anatomic endoscopic comparison of the external versus the internal approach. Plastic and Reconstructive Surgery 2003, 24612462.

9. Giacomarra V, Russolo M, Arnez ZM, Tirelli G. External osteotomy in Rhinoplasty, The laryngoscope 2001, 433438.

10. Kara CO, Kara IG, Tpuz B. Does creating a subperiosteal tunnel influence periorbital edema and ecchymosis in rhinoplasty? J oral Maxillofacial surgery 2005, 1088-1090.

11. Gryskiewicz JM, Gryskiewicz KM. Nasal osteotomies: A clinical comparison of the perforating methods versus the continuous technique. Plast Reconstructive Surgery 2002, 1445-1456. 